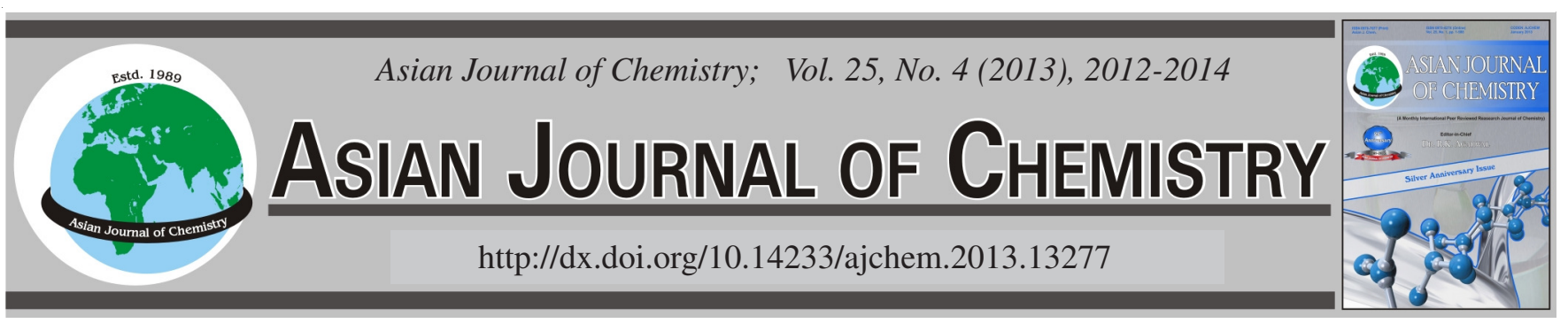

\title{
Flavonoids from Tartary Buckwheat Seeds
}

\author{
BAIXING WANG ${ }^{1}$ and HaOBIN $\mathrm{Hu}^{2, *}$
}

${ }^{1}$ College of Agriculture and Forestry, Longdong University, Qingyang 745000, P.R. China

${ }^{2}$ College of Chemistry and Chemical Engineering, Longdong University, Qingyang 745000, P.R. China

*Corresponding author: Fax: +86 934 8632822; Tel: +86 934 8522487, E-mail: hhb-88@126.com

(Received: 17 December 2011;

Accepted: 10 October 2012)

AJC-12268

\begin{abstract}
Nine flavonoids were isolated from the seeds of tartary buckwheat. Their structures were eulcidated as quercetin (1), rutin (2), vitexin (3), orientin (4), hyperin (5), tricin-7-O- $\beta$-D-glucopyranoside (6), chrysoeriol (7), tectochrysin (8) and luteolin-7-O- $\beta$-D-glucopyranoside (9), by means of spectroscopic methods, especially by ${ }^{1} \mathrm{H}$ and ${ }^{13} \mathrm{C}$ NMR, FAB-MS and GC, as well as by chemical methods and comparison with literature data.
\end{abstract}

Key Words: Tartary buckwheat seed, Flavonoid.

\section{INTRODUCTION}

Flavonoids are a group of naturally occurring plant compounds that serve many functions e.g., antioxidant, antitumor, antiinflammatory, antituberculosis, antimalarial, antimicrobial and antiviral, etc. ${ }^{1,2}$. The seeds of tartary buckwheat (Fagopyrum tataricum Gaertn.) serve as a rich source of flavonoids and have been popularly recognized as an excellent selective antioxidant and hypolipidaemic nutrient food in China and many other countries of the world ${ }^{3}$. In order to make full use of tartary buckwheat, our recent research led to the isolation of 9 flavonoids from the seeds of tartary buckwheat.

\section{EXPERIMENTAL}

Acid hydrolysis of compounds and determination of the absolute configuration of the monosaccharide: A solution of compound (5.0 mg, 2-6 and 9) in $2 \mathrm{~mol} / \mathrm{L} \mathrm{HCl}-$ dioxane $(1: 1,1 \mathrm{~mL})$ was refluxed in a water bath at $90{ }^{\circ} \mathrm{C}$ for $2 \mathrm{~h}$. After removal of dioxane, the solution was extracted with EtOAc $(1 \mathrm{~mL} \times 3)$. The aqueous layer was neutralized by passing through an Amberlite MB-3 resin column eluted with $\mathrm{H}_{2} \mathrm{O}$, then concentrated and dried to furnish a monosaccharide residue. Then, the sugars were detected by TLC analysis $\left[\mathrm{CHCl}_{3}-\mathrm{CH}_{3} \mathrm{OH}-\mathrm{H}_{2} \mathrm{O}-\mathrm{HOAc}\right.$ (15:6:2:3), detection solution: aniline-phthalic acid] against the standard samples. The residue was dissolved in pyridine $(0.2 \mathrm{~mL})$ and then a pyridine solution $(0.3 \mathrm{~mL})$ of L-cysteine methyl ester hydrochloride $(5 \mathrm{mg})$ was added to the solution. The mixture was kept at $60^{\circ} \mathrm{C}$ for $1.5 \mathrm{~h}$, dried in vacuo and trimethylsilylated with hexamethyl- disilazane-trimethylchlorosilane (HMDS-TMCS) $(0.1 \mathrm{~mL})$ at $60{ }^{\circ} \mathrm{C}$ for $1 \mathrm{~h}$. After being partitioned between $n$-hexane $(0.5$ $\mathrm{mL})$ and $\mathrm{H}_{2} \mathrm{O}(0.5 \mathrm{~mL})$, the $n$-hexane extract was concentrated and analyzed by GC under the following conditions: HP-5 MS fused silica capillary column $(30 \mathrm{~m} \times 0.25 \mathrm{~mm}$, film thinkness $0.25 \mu \mathrm{m}$ ), column temperature at $230{ }^{\circ} \mathrm{C}$, injection temperature at $250{ }^{\circ} \mathrm{C}, \mathrm{N}_{2}$ as carrier gas. The sugars were confirmed by comparison of the retention times of their derivatives with standard samples [retention time, D-glucose (21.7 min), D-galactose (25.4 min) and L-rhamnose (19.1 min)]. The presence of L-rhamnose in $\mathbf{2}$, D-galactose in $\mathbf{5}$, D-glucose in 2, 3, 4, 6 and 9 were detected.

\section{RESULTS AND DISCUSSION}

The seeds of tartary buckwheat were collected in September 2010 from Ring County of China and extracted three times with $95 \%$ aqueous EtOH at room temperature. The extract was dissoved in $50 \%$ aqueous EtOH and extracted with petroleum ether and EtOAc. The EtOAc extract was separated by repeated column chromatography (Silica-gel, Diaion HP-20 and Sephadex LH-20) and recrystallization to give nine flavonoid compounds. On the basis of chemical (acid hydrolysis) and spectral analyses (UV, IR, MS and NMR) and comparison with the literature data, their structures were elucidated as quercetin $(\mathbf{1})^{4,5}$, rutin $(\mathbf{2})^{6,7}$, vitexin $(3)^{8}$, orientin $(4)^{9}$, hyperin $(5)^{10,11}$, tricin-7-O- $\beta$-D-glucopyranoside $(6)^{12}$, chrysoeriol $(7)^{13,14}$, tectochrysin $(\mathbf{8})^{15}$ and luteolin-7-O- $\beta$-D-glucopyranoside (9) ${ }^{16,17}$. Although quercetin, rutin, vitexin, orientin and hyperin have been previously isolated from tartary buckwheat ${ }^{18}$, this is first report on the occurrence of tricin-7-O-glucopyranoside, 


\begin{tabular}{|c|c|c|c|c|c|c|c|c|c|}
\hline \multicolumn{10}{|c|}{$\begin{array}{l}\text { TABLE- } 1 \\
{ }^{1} \mathrm{H}-\mathrm{NMR} \text { DATA OF COMPOUNDS } \mathbf{1 - 9}\left(\delta_{\mathrm{H}} \text { in DMSO- } d_{6}, 400 \mathrm{MHz}, J: \mathrm{Hz}\right)\end{array}$} \\
\hline Proton & 1 & 2 & 3 & 4 & 5 & 6 & 7 & 8 & 9 \\
\hline 3 & & & $6.76(\mathrm{~s})$ & $6.64(\mathrm{~s})$ & & $7.01(\mathrm{~s})$ & $6.88(\mathrm{~s})$ & $6.68(\mathrm{~s})$ & $6.74(\mathrm{~s})$ \\
\hline 6 & $6.18(\mathrm{~d}, 2.0)$ & $6.21(\mathrm{~d}, 2.0)$ & $6.28(\mathrm{~s})$ & $6.25(\mathrm{~s})$ & $6.19(\mathrm{~d}, 2.4)$ & $6.92(\mathrm{~d}, 1.9)$ & $6.22(\mathrm{~d}, 2.0)$ & $6.39(\mathrm{~d}, 2.1)$ & $6.43(\mathrm{~d}, 1.9)$ \\
\hline 8 & $6.38(\mathrm{~d}, 2.0)$ & $6.40(\mathrm{~d}, 2.0)$ & & & $6.41(\mathrm{~d}, 2.4)$ & $6.43(\mathrm{~d}, 1.9)$ & $6.49(\mathrm{~d}, 2.0)$ & $6.51(\mathrm{~d}, 2.1)$ & $6.79(\mathrm{~d}, 1.9)$ \\
\hline $2^{\prime}$ & $7.62(\mathrm{~d}, 2.0)$ & $7.51(\mathrm{~d}, 2.1)$ & $7.99(\mathrm{~d}, 8.1)$ & $7.48(\mathrm{~d}, 2.0)$ & $7.51(\mathrm{~d}, 1.7)$ & $7.32(\mathrm{~s})$ & $7.54(\mathrm{~m})$ & $7.85(\mathrm{~m})$ & $7.41(\mathrm{~d}, 2.1)$ \\
\hline $3^{\prime}$ & & & $6.88(\mathrm{~d}, 8.1)$ & & & & & $7.48(\mathrm{~m})$ & \\
\hline $4^{\prime}$ & & & & & & & & $7.54(\mathrm{~m})$ & \\
\hline 5 & $6.88(\mathrm{~d}, 8.1)$ & $6.82(\mathrm{~d}, 8.2)$ & $6.88(\mathrm{~d}, 8.1)$ & $6.85(\mathrm{~d}, 8.4)$ & $6.81(\mathrm{~d}, 8.4)$ & & $6.98(\mathrm{~d}, 8.4)$ & $7.48(\mathrm{~m})$ & $6.88(\mathrm{~d}, 8.0)$ \\
\hline $6^{\prime}$ & $\begin{array}{l}7.56(\mathrm{dd} \\
8.1,2.0)\end{array}$ & $\begin{array}{l}7.60(\mathrm{dd}, \\
8.2,2.1)\end{array}$ & $7.99(\mathrm{~d}, 8.1)$ & $\begin{array}{l}7.52(\mathrm{dd} \\
8.4,2.0)\end{array}$ & $\begin{array}{l}7.66(\mathrm{dd} \\
8.4,1.7)\end{array}$ & $7.32(\mathrm{~s})$ & $\begin{array}{l}7.54(\mathrm{dd} \\
8.4,2.0)\end{array}$ & $7.85(\mathrm{~m})$ & $\begin{array}{l}7.46(\mathrm{dd} \\
8.0,2.1)\end{array}$ \\
\hline $\mathrm{C}_{5}-\mathrm{OH}$ & $12.48(\mathrm{~s})$ & $12.58(\mathrm{~s})$ & $13.21(\mathrm{~s})$ & $13.16(\mathrm{~s})$ & $12.62(\mathrm{~s})$ & & $12.95(\mathrm{~s})$ & $12.71(\mathrm{~s})$ & $12.98(\mathrm{~s})$ \\
\hline $\mathrm{OCH}_{3}$ & & & & & & $3.85(\mathrm{~s})$ & $3.90(\mathrm{~s})$ & $3.89(\mathrm{~s})$ & \\
\hline Anomeric $\mathrm{H}$ & & & & & & & & & \\
\hline Glu & & $5.41(\mathrm{~d}, 7.9)$ & $4.68(\mathrm{~d}, 7.6)$ & $4.67(\mathrm{~d}, 7.8)$ & & $5.02(\mathrm{~d}, 7.4)$ & & & $5.08(\mathrm{~d}, 7.5)$ \\
\hline Rha & & 4.38 (br s) & & & & & & & \\
\hline Gal & & & & & $5.36(\mathrm{~d}, 7.8)$ & & & & \\
\hline
\end{tabular}

TABLE-2

${ }^{13} \mathrm{C}-\mathrm{NMR}$ (DEPT) DATA OF COMPOUNDS 1-9 $\left(\delta_{\mathrm{C}}\right.$ in DMSO- $\left.d_{6}, 100 \mathrm{MHz}\right)$

\begin{tabular}{|c|c|c|c|c|c|c|c|c|c|c|}
\hline Carbon & 1 & \multicolumn{2}{|c|}{2} & 3 & 4 & 5 & 6 & 7 & 8 & 9 \\
\hline 2 & $146.8(\mathrm{C})$ & \multicolumn{2}{|c|}{$156.6(\mathrm{C})$} & $163.9(\mathrm{C})$ & $164.1(\mathrm{C})$ & $156.2(\mathrm{C})$ & $164.5(\mathrm{C})$ & $163.8(\mathrm{C})$ & $163.8(\mathrm{C})$ & $164.3(\mathrm{C})$ \\
\hline 3 & $135.6(\mathrm{C})$ & \multicolumn{2}{|c|}{$133.4(\mathrm{C})$} & $102.4(\mathrm{CH})$ & $102.3(\mathrm{CH})$ & $133.8(\mathrm{C})$ & $103.9(\mathrm{CH})$ & $103.8(\mathrm{CH})$ & $104.3(\mathrm{CH})$ & $103.4(\mathrm{CH})$ \\
\hline 4 & $175.6(\mathrm{C})$ & \multicolumn{2}{|c|}{$177.2(\mathrm{C})$} & $182.1(\mathrm{C})$ & $182.0(\mathrm{C})$ & $177.6(\mathrm{C})$ & $182.1(\mathrm{C})$ & $181.9(\mathrm{C})$ & $182.2(\mathrm{C})$ & $181.9(\mathrm{C})$ \\
\hline 5 & $160.1(\mathrm{C})$ & \multicolumn{2}{|c|}{$161.2(\mathrm{C})$} & $161.1(\mathrm{C})$ & $160.5(\mathrm{C})$ & $161.0(\mathrm{C})$ & $157.3(\mathrm{C})$ & $157.4(\mathrm{C})$ & $162.0(\mathrm{C})$ & $159.8(\mathrm{C})$ \\
\hline 6 & $99.0(\mathrm{CH})$ & \multicolumn{2}{|c|}{$98.7(\mathrm{CH})$} & $98.2(\mathrm{CH})$ & $98.1(\mathrm{CH})$ & $98.9(\mathrm{CH})$ & $95.4(\mathrm{CH})$ & $98.8(\mathrm{CH})$ & $98.1(\mathrm{CH})$ & $99.5(\mathrm{CH})$ \\
\hline 7 & $163.7(\mathrm{C})$ & \multicolumn{2}{|c|}{$164.1(\mathrm{C})$} & $162.6(\mathrm{C})$ & $162.6(\mathrm{C})$ & $164.3(\mathrm{C})$ & $163.2(\mathrm{C})$ & $164.2(\mathrm{C})$ & $165.2(\mathrm{C})$ & $164.3(\mathrm{C})$ \\
\hline 8 & $93.2(\mathrm{CH})$ & \multicolumn{2}{|c|}{$93.6(\mathrm{CH})$} & $104.6(\mathrm{C})$ & $104.6(\mathrm{C})$ & $93.7(\mathrm{CH})$ & $99.5(\mathrm{CH})$ & $94.6(\mathrm{CH})$ & $92.6(\mathrm{CH})$ & $94.5(\mathrm{CH})$ \\
\hline 9 & $156.0(\mathrm{C})$ & \multicolumn{2}{|c|}{$156.5(\mathrm{C})$} & $156.2(\mathrm{C})$ & $156.0(\mathrm{C})$ & $156.9(\mathrm{C})$ & $161.2(\mathrm{C})$ & $161.7(\mathrm{C})$ & $157.4(\mathrm{C})$ & $157.3(\mathrm{C})$ \\
\hline 10 & $102.5(\mathrm{C})$ & \multicolumn{2}{|c|}{$104.1(\mathrm{C})$} & 104.2 (C) & $104.1(\mathrm{C})$ & $104.0(\mathrm{C})$ & $105.4(\mathrm{C})$ & $103.3(\mathrm{C})$ & $105.6(\mathrm{C})$ & $105.2(\mathrm{C})$ \\
\hline $1^{\prime}$ & $121.8(\mathrm{C})$ & \multicolumn{2}{|c|}{$121.3(\mathrm{C})$} & $121.6(\mathrm{C})$ & $122.0(\mathrm{C})$ & $121.3(\mathrm{C})$ & $120.5(\mathrm{C})$ & $120.4(\mathrm{C})$ & $131.5(\mathrm{C})$ & $121.0(\mathrm{C})$ \\
\hline 2 ' & $114.9(\mathrm{CH})$ & \multicolumn{2}{|c|}{$115.7(\mathrm{CH})$} & $129.1(\mathrm{CH})$ & $114.0(\mathrm{CH})$ & $115.4(\mathrm{CH})$ & $104.6(\mathrm{CH})$ & $110.2(\mathrm{CH})$ & $126.2(\mathrm{CH})$ & $113.7(\mathrm{CH})$ \\
\hline $3^{\prime}$ & $144.8(\mathrm{C})$ & \multicolumn{2}{|c|}{$144.8(\mathrm{C})$} & $115.9(\mathrm{CH})$ & $145.8(\mathrm{C})$ & $145.0(\mathrm{C})$ & $148.3(\mathrm{C})$ & $150.8(\mathrm{C})$ & $129.0(\mathrm{CH})$ & $145.6(\mathrm{C})$ \\
\hline $4^{\prime}$ & 147.7 (C) & \multicolumn{2}{|c|}{$148.4(\mathrm{C})$} & $160.4(\mathrm{C})$ & 149.7 (C) & $148.6(\mathrm{C})$ & 140.1 (C) & 147.9 (C) & $131.4(\mathrm{CH})$ & $149.9(\mathrm{C})$ \\
\hline 5, & $115.4(\mathrm{CH})$ & \multicolumn{2}{|c|}{$121.7(\mathrm{CH})$} & $115.9(\mathrm{CH})$ & $115.7(\mathrm{CH})$ & $116.1(\mathrm{CH})$ & $148.3(\mathrm{C})$ & $115.8(\mathrm{CH})$ & $129.0(\mathrm{CH})$ & $115.8(\mathrm{CH})$ \\
\hline 6 ' & $119.6(\mathrm{CH})$ & \multicolumn{2}{|c|}{$116.5(\mathrm{CH})$} & $129.1(\mathrm{CH})$ & $119.4(\mathrm{CH})$ & $122.4(\mathrm{CH})$ & $104.6(\mathrm{CH})$ & $121.8(\mathrm{CH})$ & $126.2(\mathrm{CH})$ & $119.3(\mathrm{CH})$ \\
\hline \multicolumn{2}{|l|}{ Sugar } & Glu & Rha & Glu & Glu & Gal & Glu & & & Glu \\
\hline \multicolumn{2}{|l|}{1} & $101.2(\mathrm{CH})$ & $100.8(\mathrm{CH})$ & $73.8(\mathrm{CH})$ & $73.4(\mathrm{CH})$ & $101.9(\mathrm{CH})$ & $100.8(\mathrm{CH})$ & & & $100.1(\mathrm{CH})$ \\
\hline \multicolumn{2}{|l|}{2} & $74.3(\mathrm{CH})$ & $70.1(\mathrm{CH})$ & $70.8(\mathrm{CH})$ & $70.8(\mathrm{CH})$ & $71.3(\mathrm{CH})$ & $73.3(\mathrm{CH})$ & & & $73.1(\mathrm{CH})$ \\
\hline \multicolumn{2}{|l|}{3} & $76.3(\mathrm{CH})$ & $70.6(\mathrm{CH})$ & $78.6(\mathrm{CH})$ & $78.8(\mathrm{CH})$ & $73.4(\mathrm{CH})$ & $77.4(\mathrm{CH})$ & & & $77.5(\mathrm{CH})$ \\
\hline \multicolumn{2}{|l|}{4} & $70.1(\mathrm{CH})$ & $71.9(\mathrm{CH})$ & $70.5(\mathrm{CH})$ & $70.7(\mathrm{CH})$ & $68.1(\mathrm{CH})$ & $69.8(\mathrm{CH})$ & & & $69.7(\mathrm{CH})$ \\
\hline \multicolumn{2}{|l|}{5} & $75.8(\mathrm{CH})$ & $68.2(\mathrm{CH})$ & $81.7(\mathrm{CH})$ & $82.0(\mathrm{CH})$ & $75.8(\mathrm{CH})$ & $76.6(\mathrm{CH})$ & & & $76.4(\mathrm{CH})$ \\
\hline \multicolumn{2}{|l|}{6} & $67.0\left(\mathrm{CH}_{2}\right)$ & $17.5\left(\mathrm{CH}_{3}\right)$ & $61.3\left(\mathrm{CH}_{2}\right)$ & $61.7\left(\mathrm{CH}_{2}\right)$ & $60.3\left(\mathrm{CH}_{2}\right)$ & $60.8\left(\mathrm{CH}_{2}\right)$ & & & $60.6\left(\mathrm{CH}_{2}\right)$ \\
\hline
\end{tabular}<smiles>[R]c1cc(-c2oc3c([R])c([R])cc(O)c3c(=O)c2[R])cc([R])c1[R5]</smiles>

\begin{tabular}{cllllll}
\hline Flavonoid & $\mathrm{R}_{1}$ & $\mathrm{R}_{2}$ & $\mathrm{R}_{3}$ & $\mathrm{R}_{4}$ & $\mathrm{R}_{5}$ & $\mathrm{R}_{6}$ \\
\hline $\mathbf{1}$ & $\mathrm{OH}$ & $\mathrm{H}$ & $\mathrm{OH}$ & $\mathrm{OH}$ & $\mathrm{OH}$ & $\mathrm{H}$ \\
$\mathbf{2}$ & $\mathrm{OGlc}-(6 \rightarrow 1)-\mathrm{Rha}$ & $\mathrm{H}$ & $\mathrm{OH}$ & $\mathrm{OH}$ & $\mathrm{OH}$ & $\mathrm{H}$ \\
$\mathbf{3}$ & $\mathrm{H}$ & $\mathrm{Glc}$ & $\mathrm{OH}$ & $\mathrm{H}$ & $\mathrm{OH}$ & $\mathrm{H}$ \\
$\mathbf{4}$ & $\mathrm{H}$ & $\mathrm{Glc}$ & $\mathrm{OH}$ & $\mathrm{OH}$ & $\mathrm{OH}$ & $\mathrm{H}$ \\
$\mathbf{5}$ & $\mathrm{OGal}$ & $\mathrm{H}$ & $\mathrm{OH}$ & $\mathrm{OH}$ & $\mathrm{OH}$ & $\mathrm{H}$ \\
$\mathbf{6}$ & $\mathrm{H}$ & $\mathrm{H}$ & $\mathrm{OGlc}$ & $\mathrm{OCH}_{3}$ & $\mathrm{OH}$ & $\mathrm{OCH}_{3}$ \\
$\mathbf{7}$ & $\mathrm{H}$ & $\mathrm{H}$ & $\mathrm{OH}$ & $\mathrm{OCH}_{3}$ & $\mathrm{OH}$ & $\mathrm{H}$ \\
$\mathbf{8}$ & $\mathrm{H}$ & $\mathrm{H}$ & $\mathrm{OCH}_{3}$ & $\mathrm{H}$ & $\mathrm{H}$ & $\mathrm{H}$ \\
$\mathbf{9}$ & $\mathrm{H}$ & $\mathrm{H}$ & $\mathrm{OGlc}$ & $\mathrm{OH}$ & $\mathrm{OH}$ & $\mathrm{H}$ \\
\hline
\end{tabular}

chrysoeriol, tectochrysin and luteolin-7-O-glucopyranoside in this genus.

Quercetin (1), yellow needles $\left(\mathrm{CHCl}_{3}-\mathrm{MeOH}\right)$, m.p. 312$314{ }^{\circ} \mathrm{C}$. UV spectrum (MeOH, $\left.\lambda_{\max }, \mathrm{nm}\right): 208 \mathrm{sh}, 255,284$, $379 \mathrm{sh}$; IR spectrum $\left(\mathrm{KBr}, \nu_{\max }, \mathrm{cm}^{-1}\right): 3406(\mathrm{OH}), 1665(\mathrm{C}=\mathrm{O})$, 1611, 1562, 1522, 1452 (Ar); negative FAB-MS m/z: 301 [M$\mathrm{H}]^{-} .{ }^{1} \mathrm{H}$ and ${ }^{13} \mathrm{C}$ NMR (Tables 1 and 2).

Rutin (2), yellow powder ( $\left.\mathrm{CHCl}_{3}-\mathrm{MeOH}\right)$, m.p. 191-192 ${ }^{\circ} \mathrm{C}$. UV spectrum (MeOH, $\left.\lambda_{\max }, \mathrm{nm}\right): 207,259$ sh, 261, $356 \mathrm{sh}$; IR spectrum $\left(\mathrm{KBr}, \mathrm{v}_{\max }, \mathrm{cm}^{-1}\right): 3422(\mathrm{OH}), 1656(\mathrm{C}=\mathrm{O}), 1604$, 1567, 1506, 1456 (Ar), $1363\left(\mathrm{CH}_{3}\right), 1064,1025$ (glycosidic bond); negative FAB-MS m/z: $609[\mathrm{M}-\mathrm{H}]]^{-} .{ }^{1} \mathrm{H}$ and ${ }^{13} \mathrm{C}$ NMR (Tables 1 and 2).

Vitexin (3), yellow crystal (MeOH), m.p. $269-271^{\circ} \mathrm{C}$. UV $\left(\mathrm{MeOH}, \lambda_{\max }, \mathrm{nm}\right): 250,267 \mathrm{sh}, 337 \mathrm{sh}$; IR spectrum $\left(\mathrm{KBr}, v_{\max }\right.$, $\left.\mathrm{cm}^{-1}\right): 3278(\mathrm{OH}), 1660(\mathrm{C}=\mathrm{O}), 1608,1500(\mathrm{Ar})$; negative FABMS $m / z: 431[\mathrm{M}-\mathrm{H}]]^{-} .{ }^{1} \mathrm{H}$ and ${ }^{13} \mathrm{C}$ NMR (Tables 1 and 2). 
Orientin (4), yellow crystal (MeOH), m.p. 261-263 ${ }^{\circ} \mathrm{C}$. $\mathrm{UV}\left(\mathrm{MeOH}, \lambda_{\max }, \mathrm{nm}\right): 248 \mathrm{sh}, 258 \mathrm{sh}, 324$; IR spectrum $(\mathrm{KBr}$, $\left.v_{\max }, \mathrm{cm}^{-1}\right): 3312(\mathrm{OH}), 1658(\mathrm{C}=\mathrm{O}), 1612,1500(\mathrm{Ar})$; negative FAB-MS $m / z$ : $447[\mathrm{M}-\mathrm{H}]^{-} .{ }^{1} \mathrm{H}$ and ${ }^{13} \mathrm{C}$ NMR (Tables 1 and 2).

Hyperin (5), pale yellow needles (MeOH), m.p. 235-237 ${ }^{\circ} \mathrm{C}$. UV spectrum $\left(\mathrm{MeOH}, \lambda_{\max }, \mathrm{nm}\right): 206 \mathrm{sh}, 257,299,362 \mathrm{sh}$; IR spectrum $\left(\mathrm{KBr}, v_{\max }, \mathrm{cm}^{-1}\right): 3423(\mathrm{OH}), 1661(\mathrm{C}=\mathrm{O}), 1604$, 1562, 1501, 1463 (Ar); negative FAB-MS m/z: 463 [M-H] $]^{-}$. ${ }^{1} \mathrm{H}$ and ${ }^{13} \mathrm{C}$ NMR (Tables 1 and 2).

Tricin-7-O- $\boldsymbol{\beta}$-D-glucopyranoside (6), yellow needles $(\mathrm{MeOH})$, m.p. $186-188^{\circ} \mathrm{C}$. UV spectrum $\left(\mathrm{MeOH}, \lambda_{\max }, \mathrm{nm}\right)$ : 250, $266 \mathrm{sh}, 352 \mathrm{sh}$; IR spectrum $\left(\mathrm{KBr}, \nu_{\max }, \mathrm{cm}^{-1}\right): 3447(\mathrm{OH})$, $1650(\mathrm{C}=\mathrm{O}), 1607,1510,1478$ (Ar); negative FAB-MS m/z: $491[\mathrm{M}-\mathrm{H}]]^{-} .{ }^{1} \mathrm{H}$ and ${ }^{13} \mathrm{C}$ NMR (Tables 1 and 2).

Chrysoeriol (7), yellow needles, m.p. $323-325{ }^{\circ} \mathrm{C}$. UV spectrum $\left(\mathrm{MeOH}, \lambda_{\max }, \mathrm{nm}\right): 247 \mathrm{sh}, 267,345 \mathrm{sh}$; IR spectrum $\left(\mathrm{KBr}, \mathrm{v}_{\max }, \mathrm{cm}^{-1}\right): 3356(\mathrm{OH}), 1652(\mathrm{C}=\mathrm{O}), 1626,1597,1508$ (Ar); negative FAB-MS m/z: $299[\mathrm{M}-\mathrm{H}]-.{ }^{-} \mathrm{H}$ and ${ }^{13} \mathrm{C}$ NMR (Tables 1 and 2).

Tectochrysin (8), pale yellow needles (EtOH), m.p. 162$165^{\circ} \mathrm{C}$. UV spectrum (MeOH, $\left.\lambda_{\max }, \mathrm{nm}\right): 248,269 \mathrm{sh}, 312 \mathrm{sh}$; IR spectrum $\left(\mathrm{KBr}, \lambda_{\max }, \mathrm{cm}^{-1}\right): 3441(\mathrm{OH}), 1620(\mathrm{C}=\mathrm{O}), 1615$, 1595 (Ar); negative FAB-MS $m / z: 267[\mathrm{M}-\mathrm{H}]^{-} .{ }^{1} \mathrm{H}$ and ${ }^{13} \mathrm{C}$ NMR (Tables 1 and 2).

Luteolin-7-O- $\boldsymbol{\beta}$-D-glucopyranoside (9), yellow crystal (MeOH), m.p. $252-254{ }^{\circ} \mathrm{C}$. UV spectrum $\left(\mathrm{MeOH}, \lambda_{\max }, \mathrm{nm}\right)$ : $255 \mathrm{sh}, 267,351 \mathrm{sh}$; IR spectrum $\left(\mathrm{KBr}, \mathrm{v}_{\max }, \mathrm{cm}^{-1}\right): 3375(\mathrm{OH})$, 1665 (C=O), 1560, 1510 (Ar), 1095, 1030 (glycosidic bond); negative FAB-MS $m / z$ : $447[\mathrm{M}-\mathrm{H}]^{-} .{ }^{1} \mathrm{H}$ and ${ }^{13} \mathrm{C}$ NMR (Tables 1 and 2).

\section{ACKNOWLEDGEMENTS}

This work was financially supported by the Scientific and Technological project of Qingyang City (GK061-25) of China.

\section{REFERENCES}

1. S.-J. Kim, I.S.M. Zaidul, T. Suzuki, Y. Mukasa, N. Hashimoto, S. Takigawa, T. Noda, C. Matsuura-Endo and H. Yamauchi, Food Chem., 110, 814 (2008).

2. G.Y. Mechikova, T.A. Stepanova, A.I. Kalinovskii, L.P. Ponomarenko and V.A. Stonik, Chem. Nat. Comp., 44, 100 (2008).

3. Y.P. Yao, C.R. Tian and W. Cao, Agr. Sci. China, 7, 356 (2008).

4. F.Z. Chen, Y.H. He, L.S. Ding and M.K. Wang, Acta Pharm. Sin., 34, 454 (1999).

5. I. Ichemesova and A.L. Budantsev, Khim. Prir. Soedin., 287 (1994).

6. Z.H. Zhou and C.R, Yang, Acta Bot. Yunn., 22, 343 (2000).

7. J. Wang, H. Yang, Z.W. Lin, H.D. Sun, J. Wang, H. Yang, Z.W. Lin and H.D. Sun, Phytochemistry, 46, 1275 (1997).

8. B.-G. Esterdahl, Acta Chem. Scand., 33B, 400 (1979).

9. T. Kato and Y. Monrita, Chem. Pharm. Bull., 38, 2277 (1990).

10. T. Hatano, T. Yasuhara and R. Yoshihara, Planta Med., 57, 83 (1991).

11. Y. Feng, X.J. Meng and J.G. Wang, Food Sci., 28, 298 (2007).

12. Y. Hirai, S. Sanada, Y. Ida and J. Shoji, Chem. Pharm. Bull., 34, 82 (1986).

13. Y.Z. Chen, H.D. Zhang and S.M. Zhang, Chem. J. Chin. Univ., 10, 260 (1989).

14. S.V. Abdullaev, A. Sattikulov, E.K. Bairov, Y.V. Kurbatov and V.M. Malikov, Khim. Prir. Soedin., 104 (1983).

15. X.Z. Luo, J.G. Yu, L.Z. Xu, K.M. Li, P. Tan and J.D. Feng, Acta Pharm. Sin., 35, 204 (2000).

16. Y. Xu and J.Y. Liang, J. China Pharm. Univ., 36, 411 (2005).

17. J.S. Li, Y.Y. Zhao, B. Wang, X.L. Li and L.B. Ma, Acta Pharm. Sin., 31, 849 (1996).

18. V. Vito, A.-R. David, S.-C. Antonio, M. Emanuele, F.-G. Alberto and F.C. Maria, J. Cereal Sci., 52, 170 (2010). 